\title{
LECCIONES APRENDIDAS Y FUTURAS ESTRATEGIAS EMPRESARIALES A UN AÑO DE LA PANDEMIA
}

\author{
LESSONS LEARNED AND FUTURE BUSINESS STRATEGIES ONE YEAR AFTER \\ THE PANDEMIC
}

Will Erick Araujo-Aguirre Universidad de San Martín de Porres Lima, Perú

ORCID: https://orcid.org/0000-0003-1889-835X Correo electrónico: willerick.araujo@usmp.pe

\section{RESUMEN}

A un año de la declaratoria de la pandemia por el COVID-19, las empresas tuvieron que vivir un escenario de crisis y enfrentarlo a través de acciones estratégicas, dejando lecciones importantes para futuras crisis. Este trabajo tuvo por objetivo describir y responder dos interrogantes importantes referidas a las prácticas empresariales y lecciones durante la pandemia, y las políticas o estrategias futuras en el escenario pospandemia. El método utilizado fue una revisión sistemática de artículos científicos, siguiendo una estrategia de búsqueda bibliográfica en la base de datos de Scopus y utilizando algunos criterios como filtros para refinar la búsqueda. Los resultados mostraron dos temáticas recurrentes en el análisis; estas fueron la tecnología y la innovación, las cuales se encuentran en la gran parte de las estrategias y acciones implementadas por las empresas; así como muchas otras que se sugieren poner en práctica pospandemia, como políticas de financiamiento, abastecimiento, entre otras. Algunas limitaciones que se tuvieron fueron el uso de un solo tipo de fuente científica, pocas áreas temáticas y una base de datos reconocida para la búsqueda y análisis.

Palabras clave: COVID-19; pospandemia; crisis; estrategias; empresas.

\begin{abstract}
One year after the COVID-19 pandemic, companies had to experience the crisis scenario and overcome it through strategic actions, which left important lessons for the possibility of future crises. The objective of this research was to describe and answer two important questions regarding business practices and lessons during the pandemic, and future policies or strategies in the post-pandemic scenario. The method used was a systematic review of scientific articles, following a bibliographic search strategy in the Scopus database, using some criteria as filters to refine the search. The results show two recurring themes found in the analysis, such as technology and innovation, which are found in most of the strategies and actions implemented by companies; as well as many others measures that are suggested to be put into practice after the end of the pandemic, such as financing and supply policies, among others. Some limitations were the use of a single type of scientific source, few subject areas, and a recognized database for search and analysis.
\end{abstract}

Keywords: COVID-19; crisis; post-pandemic; strategies; companies.

(c) Los autores. Este artículo es publicado por la revista Quipukamayoc de la Facultad de Ciencias Contables, Universidad Nacional Mayor de San Marcos. Este es un artículo de acceso abierto, distribuido bajo los términos de la licencia Creative Commons Atribución 4.0 Internacional (CC BY 4.0) [https://creativecommons.org/licenses/ by/4.0/deed.es] que permite el uso, distribución y reproducción en cualquier medio, siempre que la obra original sea debidamente citada de su fuente original. 


\section{INTRODUCCIÓN}

En la tercera semana del mes de marzo de 2020, el presidente de la Organización Mundial de la Salud (OMS) declaró la rápida expansión del COVID-19 como una pandemia. A partir de aquel momento, los gobiernos de cada país tomaron medidas y políticas sanitarias para frenar la expansión del COVID-19 en sus territorios. Una de las medidas más adoptadas por todos los países fue la cuarentena, la cual significó la inmovilización de las personas, limitando las actividades comerciales de todos los sectores y solo permitiendo el funcionamiento de las actividades esenciales relacionadas con la salud, limpieza pública, entre otras.

Con relación a lo anterior, Dai et al. (2021) explican que el COVID-19 produjo un gran impacto en las pequeñas y microempresas (pymes) chinas, que se derivaron en efectos sustanciales entre varios sectores y regiones de dicho país. Por otro lado, las acciones para afrontar el COVID-19, como fueron las medidas de restricción y el distanciamiento social, provocaron que el comportamiento del consumidor cambiara (Sheth, 2020b), ampliando el consumo en el comercio electrónico desde la compra de productos, pasando a los servicios de ocio y diversión hasta los pedagógicos (Lechuga Nevárez y Hernández Chavarria, 2020). Es decir, la pandemia provocada por el COVID-19 y las formas de enfrentarla tuvieron repercusiones en los negocios y en los hábitos de compra de los consumidores.

No obstante, hay que rescatar que los efectos del COVID-19 no fueron del todo malos o perjudiciales para las empresas o para los futuros emprendedores. Para ser más específicos, en México, jóvenes emprendedores decidieron enfrentar a la pandemia aún con limitaciones y escasos recursos a su disposición (Montiel Méndez, Flores Novelo, Avila Paz y Sierra Martinez 2021). Asimismo, Kuckertz et al. (2020) mencionan que los empresarios, dentro de su entorno, ven a la oportunidad empresarial como una forma positiva de enfrentar problemas de la actualidad a través de una estrategia empresarial. Además, que se espera que sean flexibles y adapten sus modelos comerciales para reaccionar a una crisis. De igual modo, Ding y Li (2021) observan que la pandemia podría constituir una oportunidad para que se produzca las adquisiciones y fusiones comerciales. También aseguran que la oportunidad se encuentra en la supervivencia de las empresas en el corto plazo, con una expectativa de crecimiento en el largo plazo. Lo que significa que, a pesar de que se encuentren dificultades, también se puede aprender y obtener lecciones importantes de la crisis actual como sucedió en épocas anteriores.

En ese contexto, el objetivo de este trabajo es describir las decisiones, estrategias y lecciones empresariales aprendidas dentro del contexto de la pandemia originada por el
COVID-19. Además, exponer las implicaciones futuras que estas enfrentarán, teniendo en cuenta que al momento de redactarse el presente trabajo se cumplió un año de haberse declarado la pandemia mundial por la OMS. De modo que, para alcanzar el objetivo planteado, se realizó un análisis sistemático de resultados de investigación de artículos científicos, respondiendo a las preguntas de investigación: (1) ¿Cuáles fueron las prácticas empresariales y lecciones en la pandemia?, y (2) ¿cuáles son las políticas o estrategias en el escenario pospandémico?

El análisis de la literatura en este estudio utilizó el método de revisión sistemática para una eficiente búsqueda bibliográfica. La base de datos usada fue Scopus y Google Schoolar. Este último, sirvió como valor agregado para enriquecer la búsqueda en el estudio, ya que Martín-Martín, Orduna-Malea, Thelwall y Delgado López-Cózar (2018) mencionan que existe la certeza de que Google Schoolar ubica de modo relevante más citas que la colección principal de Web of Sciencie y Scopus en diversos temas.

El trabajo de revisión inició con la selección de las palabras clave como "enterprise", "business", “COVID-19” y "pandemic", con la finalidad de resaltar los artículos que se relacionen con las preguntas de investigación y el objetivo principal.

En segundo lugar, situados en las bases de datos respectivas, se aplicaron los filtros de búsqueda con la intención de refinar aún más la investigación. Los filtros tuvieron las categorías como el año de publicación, tipo de artículo y área temática. Los años de publicación que se usaron fueron los dos últimos, teniendo en cuenta el presente año. Por otra parte, en lo que respecta al tipo de artículo, se eligieron aquellos de investigación, y las áreas temáticas seleccionadas fueron negocios, gestión, contabilidad, economía y finanzas. Finalmente, como consecuencia de la búsqueda y selección de las publicaciones revisadas, resultaron 40 artículos que sirvieron para el análisis, discusión y desarrollo de la presente investigación, adicionalmente se incluyó otras referencias para enriquecer el análisis.

Las limitaciones que tuvo el trabajo de investigación fue la base de datos seleccionada, ya que esta solo fue Scopus y, por ello, las áreas temáticas escogidas fueron escasas. De modo que se debe de tomar en cuenta una base de datos de similar rigurosidad para ampliar el análisis y las áreas temáticas podrían ser extendidas a sus análogos o subtemas. Por último, los tipos de artículos que se usaron solo fueron artículos científicos; el análisis de revisiones, resúmenes de conferencias, reportes de caso, entre otros, ayudarían a mejorar de forma sustancial futuras revisiones. 


\section{ALCANCE DE LA REVISIÓN}

\section{Los problemas de las empresas en el escenario de la pandemia}

\section{Impacto productivo y económico}

El escenario de la pandemia tuvo diferentes efectos en las empresas del mundo, por ejemplo, la Comisión Económica para América Latina y el Caribe (2020) señala que la crisis que generó el COVID-19 arremetió contra una estructura organizacional con debilidades de décadas pasadas. Además, precisa datos importantes del impacto de la pandemia para el primer cuatrimestre de 2020 en comparación con el año 2019, como la situación en la región de Brasil donde la producción en el sector industrial cayó un $8.2 \%$, en México un 10.9\%, Argentina un 13.5\% y probablemente el más afectado fue Perú con reducción del $21.4 \%$. En ese contexto, todos estos datos reflejaban que las empresas tendrían problemas con respecto a sus modelos de negocio en general, desde lo logístico, ventas, producción, entre otras.

En relación con lo anterior, Araújo Vila (2020) menciona que los sectores del turismo y el comercio han tenido mayores dificultades. Asimismo, sostiene que los problemas son a causa de que dichas actividades empresariales tenían deficiencias en su planificación de gestión de la crisis. Es importante mencionar que, estas dificultades en la gestión y planificación se suman a una economía en el mundo con un desempeño débil antes de la pandemia por el COVID-19 (Comisión Económica para América Latina y el Caribe, 2020a). En ese sentido, este impacto económico se reflejaría en factores y problemas estructurales dentro de las empresas, con respecto a sus procesos de negocio y cadena de valor, como la infraestructura, logística, tecnología, entre otros.

\section{Procesos organizacionales}

Considerando el impacto del COVID-19, Thorgren y Williams (2020) explican que las pymes tomaron decisiones extremas en respuesta a la crisis, como reducir su flujo de caja negativo con la probabilidad de declararse en quiebra, en reemplazo de reforzar la relación entre la deuda y el capital con el fin de evitar problemas financieros futuros. Por otra parte, Meyer, Prescott y Sheng (2020), en sus resultados, encontraron que las empresas vieron a la crisis pandémica como un estado que produjo más demanda que oferta. Adicionalmente, en respuesta al impacto de la demanda, tuvieron que reducir los sueldos de gran parte sus trabajadores, proyectando recortes más sustanciales a finales del año 2020 y adelantando la disminución de los precios de venta en un corto plazo. Es decir, las decisiones empresariales frente a la crisis supusieron una situación muy complicada, con iniciativas difíciles y arriesgadas para subsistir en la situación pandémica.
De manera semejante, Tang, Chin y Lee (2021) descubrieron que a consecuencia del COVID-19, las acciones de las empresas taiwanesas mostraron debilidad en los negocios asociados a los sistemas de cadena de suministros y con la posibilidad de anular el abastecimiento de bienes y el rendimiento de acciones. Todo esto parece confirmar que además de los problemas de flujos negativos y demanda de la oferta mencionados en el párrafo anterior, la cadena de suministro y las acciones se vieron afectadas de igual forma. Asimismo, la pandemia modificó de forma sustancial el entorno empresarial dejando a la mayoría de negocios vigilantes ante las pérdidas (Liguori y Pittz, 2020). En otras palabras, ninguno de estos negocios en el mundo esperaba que la crisis relacionada por las medidas para afrontar el COVID-19 afectaría su productividad de manera tan repentina y con un impacto mayor de lo que pensaban o que serían capaces de sostener en un corto plazo.

\section{Las lecciones aprendidas en pandemia}

\section{Medidas y acciones estratégicas}

Todos los sectores de negocios estaban poco preparados, sin un plan de contingencia o estrategias para asumir retos de crisis globales en general. Hao, Xiao y Chon (2020) explican que, a pesar de que la industria hotelera en China fue una de las primeras en tener contacto con la pandemia, adoptó medidas ágiles para atenuar los prejuicios económicos como la protección a sus empleados y clientes. A su vez, Nguyen, Ngo y Tran (2021) encontraron que las empresas vietnamitas tenían un pensamiento estratégico, sólido y estaban preparadas para enfrentar una crisis como la originada por el COVID-19. Por su parte, Ramírez Charca y Campos Lizarzaburu (2020) identificaron medidas de actuación en las micro y pequeñas empresas (MYPE) peruanas, relacionadas con un nivel estratégico, como las estrategias de enfoque, el desarrollo y retirada organizacional; y, en el nivel operacional se destacaron el incremento de la cartera de clientes, presencia en la red y el cambio del giro empresarial.

Por otra parte, Breier et al. (2021) sostienen que el Modelo Innovación Empresarial (BMI, en inglés) es una estrategia valiosa para que las empresas del sector hotelero hagan frente y reanuden sus actividades después de la crisis de la pandemia. Mientras que, de modo idéntico, Lee y Trimi (2021) señalan que la convergencia de innovación puede ser una herramienta clave para manejar situaciones relacionadas con las crisis pandémicas mundiales. Sin embargo, según Caballero-Morales (2021), para poner en marcha la innovación se debe de llevar a cabo una optimización de los procesos productivos de las empresas. En otras palabras, la innovación en sus distintos modelos es otra de las alternativas para sobrellevar la actual situación de crisis, pero necesita de condiciones óptimas en los procesos de negocios dentro de las empresas. 


\section{Cadena de suministro y operaciones}

Tomando como referencia el punto anterior, se observa que la pandemia sacó a relucir las debilidades empresariales tanto internas como externas. Teniendo en cuenta ello, Tang et al. (2021) mencionan que para prevenir los riesgos de la interrupción de la cadena de suministro, el punto más importante es observar a la logística de la empresa, regresando al país de origen o multiplicar las ubicaciones de producción con el fin de minimizar el impacto en un brote epidémico. Por otra parte, Björklund, Mikkonen, Mattila, y van der Marel (2020) explica que las empresas, a través del empleo de la experimentación, disminuyen los efectos de la crisis ocasionada por la pandemia, además de ampliar su universo de soluciones y capacidad de aprovecharlas. Esto significa que, alternativas como la prevención en el caso de la cadena suministro y la experimentación, son medidas que deben tenerse en cuenta cuando las empresas enfrentan crisis como el COVID-19.

Por su parte, Sheth (2020a) muestra cómo las empresas industriales pueden sobrevivir a situaciones como la pandemia o incluso más extremas, a través de las siguientes acciones: a) orientar la acción a los stakeholders, b) medir la satisfacción de los stakeholders, c) convertirse en un buen cliente, d) convertirse en asesores confiables, e) modernizar las tecnologías de la información, e f) incorporar un propósito a la empresa.

Cabe indicar también que autores como Brown y Rocha (2020) mencionan que para solucionar los problemas asociados al distanciamiento financiero ocasionado por el COVID-19, la alternativa para los formuladores de políticas, inversores y empresarios sería la participación en línea en plataformas similares a las redes online de financiación que utilizan los privados. Mientras que, en lo que respecta al distanciamiento en relación con las ventas, Papadopoulos, Baltas y Balta (2020) afirman que, pospandemia, las pymes deberían de incorporar en sus estrategias de negocio los escenarios de crisis y planes empresariales de continuidad en la medida que usen medios alternativos de venta, buscando el aumento de sus ingresos. Dicho de otra manera, las alternativas que calzan con el distanciamiento de las finanzas se integran con plataformas en línea para buscar mayor participación; en cambio, en las ventas, las crisis deben ser un factor de riesgo para dicha actividad, además de que se debe buscar medios alternativos para seguir obteniendo ingresos mientras esté en marcha alguna crisis.

\section{Transformación digital}

Como un punto importante en las lecciones aprendidas en la pandemia se tiene a la transformación digital. En este sentido, Palomino Pita, Mendoza Vargas y Oblitas
Cruz (2020) señalan que los negocios tienen que adoptar el comercio electrónico para establecer una relación con sus clientes y satisfacer sus necesidades para provocar una nueva compra. No obstante, Useche Aguirre, Salazar Vázquez, Barragán Ramírez y Sánchez Salazar (2020) mencionan que todas las estrategias más allá de su posición deben buscar el desarrollo de capacidades empresariales, y que el gestor estratégico debe estar en armonía con las herramientas de tecnologías de la información y comunicación (TIC) para lograr disminuir brechas en las inversiones TIC y habilidades de los empleados. Es decir, las acciones en conjunto con las estrategias que se adopten como desafío deben de entenderse como capacidades que no trabajan aisladas de otras; sino que, se debe de buscar congruencia entre las acciones, estrategias e ideas en conjunto con los estrategas y las tecnologías de vanguardia.

Es preciso subrayar que, cuando se habla de transformación digital en la década actual, también se hace referencia a las nuevas tendencias de las tecnologías empresariales de vanguardia para mejorar las organizaciones como la Inteligencia Artificial, Internet de las cosas, Blockchain, entre otras. Dicho esto, Polas y Raju (2021) mencionan que la tecnología esta dominando las actividades de entrega de bienes y servicios, y es indudable que la inteligencia artificial facilitará su crecimiento, así como crear nuevas oportunidades en los negocios y ventajas competitivas. Por otro lado, Frizzo-Barker et al. (2020) señalan que la tecnología Blockchain está creciendo de forma rápida en un contexto de incertidumbre. Además, mencionan que puede extenderse su aplicación más allá del sector financiero, con una práctica empresarial en general. Lo que parece confirmar lo referido en el párrafo anterior, sobre la importancia de las tecnologías de información para el desarrollo de las organizaciones, juntamente con capacidades humanas. Es decir, lo mismo sucede con las tecnologías empresariales que son tendencia actualmente, con su manera de sobrellevar y brindar un mayor valor a las empresas en el contexto actual.

\section{El escenario empresarial pospandémico}

Lo dicho hasta aquí supone que hubo dos grandes grupos empresariales que enfrentaban la pandemia. Por una parte, por ejemplo, los que tomaban medidas drásticas con el afán de poder subsistir en los mercados con acciones relacionadas a la reducción de flujo de cajas. Mientras que otro grupo de empresas, tenían idea estratégica de cómo enfrentar una crisis a través de acciones como la presencia en la red o medidas ágiles de protección de clientes y empleados. Todo esto parece confirmar que las empresas de alguna forma aprendieron algo de la pandemia, sea positivo o negativo. En este sentido, el escenario después de la pandemia ocasionada por el COVID-19, debe de tomarse como una oportunidad para desarrollar nuevas estrategias 
empresariales, políticas públicas; $y$, posiblemente, reinventar los modelos de negocios en esta nueva década hacia una nueva revolución industrial o tal vez tecnológica.

Así, por ejemplo, Ratten (2020) expone que los empresarios deben de enfocarse en las medidas políticas que resultaron de la pandemia, también considerar oportunidades novedosas empresariales. En esta misma línea, Belhadi et al. (2021) sostienen que en épocas definidas por cambios sustanciales, la cohesión entre el gobierno y la industria debe de buscar rediseñar la manera en que se hacen los negocios hacia una construcción de una manufactura sostenible, ágil y con capacidad de afrontar adversidades. Además, Shafi, Liu y Ren (2020) proponen diferentes políticas para enfrenar la crisis actual y las venideras, con el fin de mitigar la carga en las micro, pequeña y mediana empresa (MIPYMES) como: a) proteger y apoyar al empleo, b) impulsar la economía, c) la planificación, d) desarrollar las capacidades de resiliencia, y e) fomentarfomentar las relaciones sociales efectivas.

Con base en lo anterior, se notó que las políticas públicas y empresariales para enfrentar crisis serán importantes para un futuro como la pandemia actual, que se vinculan con los aspectos labores, económicos y sociales, y son temas que siempre resaltan en cualquier situación. Ahora, se considera autores como Nicolás Martínez y Rubio Bañón (2020), quienes mencionan que para que los emprendedores puedan continuar con sus operaciones, necesitan de asistencia institucional tras una crisis ocasionada por el COVID-19, pues estos deben hacer frente a retos tales como la digitalización, financiación, reducción del capital de trabajo y posibles ideas de negocio. Como menciona Katz, Jung y Callorda (2020), la digitalización es importante para enfrentar situaciones de emergencia, de manera que los países deben prevenir el desarrollo de tecnología digital con infraestructura de banda ancha fija y móvil con el propósito de hacer frente a situaciones futuras. Teniendo en cuenta que, como consecuencia del COVID-19, la tecnología digital pasó a ser reconocida y usada en actividades en el trabajo y en la vida diaria (Zahra, 2021). Aún más, en el sector minorista la tecnología ha tenido un alto impacto frente situaciones particulares y repentinas (Shankar et al., 2020). Cabe resaltar que la digitalización es importante, de modo que, apuntar la mirada a dicho factor ayudará a temas relacionados como el trabajo, la financiación y los nuevos modelos de negocio.

Por otra parte, Akpan, Udoh y Adebisi (2020) mencionan que, las oportunidades causadas por el COVID-19 apuntan a la reaparición de una nueva generación de emprendedores que lideren la nueva revolución industrial con el descubrimiento de nuevos modelos de negocios basados en tecnologías modernas. No obstante, es importante tener en cuenta que Akpan, Soopramanien y Kwak (2020) evidenciaron que las pequeñas empresas no se sienten apresuradas a adoptar y poner en funcionamiento las tecnologías de la información avanzada, muy aparte de las tecnologías comunes que usan. Sin embargo, Ebersberger y Kuckertz (2021) mencionan que desde una óptica social, los retos como consecuencia del COVID-19 aparentemente son atractivos para poder afrontarlos a través de la combinación de varios tipos de innovadores. En este sentido se comprende que, dentro del sector empresarial, la pandemia deja un camino positivo para el surgimiento de nuevas ideas de negocio; sin embargo, algunos de los empresarios ante estas circunstancias aún denotan tranquilidad con la implementación de estrategias tecnológicas que se volvieron importantes en esta crisis.

\section{CONCLUSIONES}

A lo largo del análisis, se encontraron dos temas recurrentes que diversos autores manifestaban en sus trabajos de investigación. El primero relacionado con la tecnología, la cual ha ido escalando en los negocios, educación y múltiples sectores, volviéndose casi imprescindible en las estrategias de dichos sectores, como se señaló en párrafos precedentes. Ahora, en la actual situación, la tecnología no ha pasado desapercibida, ya que como mencionan Cueva Kean Chong, Piedra Arévalo y Zarate Enriquez (2020) para sobrevivir al aislamiento social o situaciones pandémicas, las estrategias administrativas y tecnológicas son sumamente importantes. Además, algunas de las estrategias o herramientas están relacionadas con la web, redes sociales, publicidad y posicionamiento web.

Siguiendo el razonamiento anterior, el comercio electrónico aumentó porque se adicionaron estrategias como la redes en internet, uso de móviles, entre otras. Si bien es cierto, así como la tecnología y las redes web son valiosas para crisis, como la ocasionada por el COVID-19, algunos de los empresarios aún no se ven motivados totalmente o van a paso lento con su implementación, debido a limitaciones de recursos económicos o el conocimiento del mundo digital, que ya es algo preocupante en la época actual o se debe a que la mayoría de los negocios son pequeñas empresas. Sin embargo, el tamaño de la empresa no es un impedimento para la adopción de tecnología, un claro ejemplo son los pequeños negocios de venta que implementaron los pagos electrónicos con aplicaciones, los cuales no son necesariamente grandes tecnologías.

El segundo tema se relaciona con la innovación y las principales formas desarrolladas y utilizadas en pandemia como la BMI o la convergencia de la innovación. Desde innovaciones de estrategias hasta las políticas y acciones para sobreponerse de diversos riesgos empresariales. Es necesario subrayar que, para que la innovación prospere 
se debe de mirar a dos lados, el interno y el externo. Visto de esta forma, las relaciones abiertas agilizan el proceso de innovación interna, permitiendo aprovechar la información externa para fortalecer un negocio mientras utilizan nuestro conocimiento del negocio de forma similar (Chesbrough, 2020). En otras palabras, las relaciones externas fortalecen la innovación interna y viceversa, con la obtención de información de ambas partes, para que luego se convierta en estrategias empresariales.

Además de los temas anteriores, se debe mencionar también que Gunay y Kurtulmuş (2021) sugieren en su estudio que se ponga en marcha las medidas y estrategias relacionadas con el capital de trabajo, cadena de suministro y la gestión de riesgos. Esto significa que las estrategias y medidas que se mencionan serán determinantes y tal vez podrían configurar temas de estudio con respecto a sus resultados como políticas o estrategias. Adicionalmente, Amankwah-Amoah, Khan y Wood (2020) comentan que los dueños de pequeñas empresas que renuncian a las actividades empresariales como resultado del COVID-19 pasan inadvertidos, pero esto representa un motivo importante para indagar como los mismos empresarios retornan con nuevas empresas. Es decir, esto se traduce que en futuras investigaciones se tendrá que profundizar esta cuestión en particular, la cual podría ser muy interesante estudiar, así como todos los temas tratados en el presente trabajo.

Finalmente, todo lo analizado, hasta este punto, supone algunas medidas para reponerse de la crisis causada por el COVID-19. Sin embargo, el análisis presentado en este trabajo debe de tomarse como algo referencial, debido que hasta la fecha la pandemia no ha acabado, e incluso en algunos países se habla de una tercera ola de contagios por el COVID-19.

\section{REFERENCIAS}

Akpan, I. J., Soopramanien, D., \& Kwak, D. H. (2020). Cutting-edge technologies for small business and innovation in the era of COVID-19 global health pandemic. Journal of Small Business and Entrepreneurship, 1-11. https://doi.org/10.1080/08276331.2020.1799294

Akpan, I. J., Udoh, E. A. P., \& Adebisi, B. (2020). Small business awareness and adoption of state-of-the-art technologies in emerging and developing markets, and lessons from the COVID-19 pandemic. Journal of Small Business \& Entrepreneurship, 1-18. https://doi.or $\mathrm{g} / 10.1080 / 08276331.2020 .1820185$

Amankwah-Amoah, J., Khan, Z., \& Wood, G. (2020). COVID-19 and business failures: The paradoxes of experience, scale, and scope for theory and practice. European Management Journal. https://doi.org/10.1016/j.emj.2020.09.002

Araújo Vila, N. (2020). Repercusión económica de la pandemia originada por el COVID-19 a nivel mundial. Análisis de sectores más afectados. Quipukamayoc, 28(57), 85-93. https://doi.org/10.15381/quipu. v28i57.17903

Belhadi, A., Kamble, S., Jabbour, C. J. C., Gunasekaran, A., Ndubisi, N. O., \& Venkatesh, M. (2021). Manufacturing and service supply chain resilience to the COVID-19 outbreak: Lessons learned from the automobile and airline industries. Technological Forecasting and Social Change, 163, 120447. https://doi.org/10.1016/j. techfore.2020.120447

Björklund, T. A., Mikkonen, M., Mattila, P., \& van der Marel, F. (2020). Expanding entrepreneurial solution spaces in times of crisis: Business model experimentation amongst packaged food and beverage ventures. Journal of Business Venturing Insights, 14. https://doi. org/10.1016/j.jbvi.2020.e00197

Breier, M., Kallmuenzer, A., Clauss, T., Gast, J., Kraus, S., \& Tiberius, V. (2021). The role of business model innovation in the hospitality industry during the COVID-19 crisis. International Journal of Hospitality Management, 92, 102723. https://doi.org/10.1016/j. ijhm.2020.102723

Brown, R., \& Rocha, A. (2020). Entrepreneurial uncertainty during the COVID-19 crisis: Mapping the temporal dynamics of entrepreneurial finance. Journal of Business Venturing Insights, 14, e00174. https://doi.org/10.1016/j.jbvi.2020.e00174

Caballero-Morales, S.-O. (2021). Innovation as recovery strategy for SMEs in emerging economies during the COVID-19 pandemic. Research in International Business and Finance, 57, 101396. https://doi.org/10.1016/j. ribaf.2021.101396

Chesbrough, H. (2020). To recover faster from COVID-19, open up: Managerial implications from an open innovation perspective. Industrial Marketing Management, 88, 410-413. https://doi.org/10.1016/j. indmarman.2020.04.010

Comisión Económica para América Latina y el Caribe. (2020a). América Latina y el Caribe ante la pandemia del COVID-19. Efectos económicos y sociales. En Informe Especial COVID-19 (Número 1). http://hdl. handle.net/11362/45337 
Comisión Económica para América Latina y el Caribe. (2020b). Sectores y empresas frente al COVID-19: emergencia y reactivación. En Informe Especial COVID-19 (Vol. 4). http://hdl.handle.net/11362/45734

Cueva Kean Chong, T. V., Piedra Arévalo, B. F., \& Zarate Enriquez, V. A. (2020). Estrategias administrativas en tiempos de distanciamiento social y herramientas web. Polo del Conocimiento, 5(2), 916-929. https://doi. org/10.23857/pc.v5i2.1756

Dai, R., Feng, H., Hu, J., Jin, Q., Li, H., Wang, R., Wang, R., Xu, L., \& Zhang, X. (2021). The impact of COVID-19 on small and medium-sized enterprises (SMEs): Evidence from two-wave phone surveys in China. China Economic Review, 67, 101607. https://doi.org/10.1016/j.chieco.2021.101607

Ding, A. W., \& Li, S. (2021). National response strategies and marketing innovations during the COVID-19 pandemic. Business Horizons, 64(2), 295-306. https:// doi.org/10.1016/j.bushor.2020.12.005

Ebersberger, B., \& Kuckertz, A. (2021). Hop to it! The impact of organization type on innovation response time to the COVID-19 crisis. Journal of Business Research, 124, 126-135. https://doi.org/10.1016/j.jbusres.2020.11.051

Frizzo-Barker, J., Chow-White, P. A., Adams, P. R., Mentanko, J., Ha, D., \& Green, S. (2020). Blockchain as a disruptive technology for business: A systematic review. International Journal of Information Management, 51, 102029. https://doi.org/10.1016/j.ijinfomgt.2019.10.014

Gunay, S., \& Kurtulmuş, B. E. (2021). COVID-19 social distancing and the US service sector: What do we learn? Research in International Business and Finance, 56, 101361. https://doi.org/10.1016/j.ribaf.2020.101361

Hao, F., Xiao, Q., \& Chon, K. (2020). COVID-19 and China's Hotel Industry: Impacts, a Disaster Management Framework, and Post-Pandemic Agenda. International Journal of Hospitality Management, 90, 102636. https://doi.org/10.1016/j.ijhm.2020.102636

Katz, R., Jung, J., \& Callorda, F. (2020). Can digitization mitigate the economic damage of a pandemic? Evidence from SARS. Telecommunications Policy, 44(10), 102044. https://doi.org/10.1016/j.telpol.2020.102044

Kuckertz, A., Brändle, L., Gaudig, A., Hinderer, S., Morales Reyes, C. A., Prochotta, A., Steinbrink, K. M., \& Berger, E. S. C. (2020). Startups in times of crisis - A rapid response to the COVID-19 pandemic. Journal of
Business Venturing Insights, 13, e00169. https://doi.org/10.1016/j.jbvi.2020.e00169

Lechuga Nevárez, M. del R., \& Hernández Chavarria, J. (2020). La primavera del 2020: Las repercusiones del COVID-19 en los patrones de consumo y el comercio electrónico en Durango, México. Revista de Economía del Caribe, 26. http://portal.amelica.org/ameli/ jatsRepo/318/3181675004/index.html

Lee, S. M., \& Trimi, S. (2021). Convergence innovation in the digital age and in the COVID-19 pandemic crisis. Journal of Business Research, 123, 14-22. https://doi.org/10.1016/j.jbusres.2020.09.041

Liguori, E. W., \& Pittz, T. G. (2020). Strategies for sma11 business: Surviving and thriving in the era of COVID-19. Journal of the International Council for Small Business, 1(2), 106-110. https://doi.org/10.1080/26437 015.2020 .1779538

Martín-Martín, A., Orduna-Malea, E., Thelwall, M., \& Delgado López-Cózar, E. (2018). Google Scholar, Web of Science, and Scopus: A systematic comparison of citations in 252 subject categories. Journal of Informetrics, 12(4), 1160-1177. https://doi.org/10.1016/j. joi.2018.09.002

Meyer, B. H., Prescott, B., \& Sheng, X. S. (2020). The Impact of the COVID-19 Pandemic on Business Expectations. SSRN Electronic Journal. https://doi.org/10.2139/ ssrn.3690489

Montiel Méndez, O. J., Flores Novelo, A., Avila Paz, E., \& Sierra Martinez, S. J. (2021). “Tengo que sobrevivir”: Relato de vida de tres jóvenes microemprendedores bajo COVID-19. Telos Revista de Estudios Interdisciplinarios en Ciencias Sociales, 23(1), 67-84. https://doi. org/10.36390/telos231.06

Nguyen, H. H., Ngo, V. M., \& Tran, A. N. T. (2021). Financial performances, entrepreneurial factors and coping strategy to survive in the COVID-19 pandemic: case of Vietnam. Research in International Business and Finance, 56, 101380. https://doi.org/10.1016/j.ribaf.2021.101380

Nicolás Martínez, C., \& Rubio Bañón, A. (2020). Emprendimiento en épocas de crisis: Un análisis exploratorio de los efectos del COVID-19. Small Business International Review, 4(2), 53-66. https://doi.org/10.26784/ sbir.v4i2.279

Palomino Pita, A. F., Mendoza Vargas, C. \& Oblitas Cruz, J. F. (2020). E-commerce and its importance in times of COVID-19 in Northern Peru. Revista Venezolana 
de Gerencia, 25(3), 253-266. https://doi.org/10.37960/ rvg.v25i3.33367

Papadopoulos, T., Baltas, K. N., \& Balta, M. E. (2020). The use of digital technologies by small and medium enterprises during COVID-19: Implications for theory and practice. International Journal of Information Management, 55, 102192. https://doi.org/10.1016/j.ijinfomgt.2020.102192

Polas, M. R. H., \& Raju, V. (2021). Technology and Entrepreneurial Marketing Decisions During COVID-19. Global Journal of Flexible Systems Management, 22(2), 95-112. https://doi.org/10.1007/s40171-021-00262-0

Ramírez Charca, E. D., \& Campos Lizarzaburu, W. B. (2020). Microempresas en pandemia: una aproximación desde el discurso del emprendedor. Economía \& Negocios, 2(2), 22-31. https://doi.org/10.33326/270860 62.2020.2.968

Ratten, V. (2020). Coronavirus (COVID-19) and entrepreneurship: changing life and work landscape. Journal of Small Business and Entrepreneurship, 1-14. https://doi. org/10.1080/08276331.2020.1790167

Shafi, M., Liu, J., \& Ren, W. (2020). Impact of COVID-19 pandemic on micro, small, and medium-sized Enterprises operating in Pakistan. Research in Globalization, 2, 100018. https://doi.org/10.1016/j.resglo. 2020.100018

Shankar, V., Kalyanam, K., Setia, P., Golmohammadi, A., Tirunillai, S., Douglass, T., Hennessey, J., Bull, J. S., \& Waddoups, R. (2020). How Technology is Changing Retail. Journal of Retailing. https://doi.org/10.1016/j. jretai.2020.10.006
Sheth, J. (2020a). Business of business is more than business: Managing during the Covid crisis. Industrial Marketing Management, 88, 261-264. https://doi.org/10.1016/j.indmarman.2020.05.028

Sheth, J. (2020b). Impact of COVID-19 on consumer behavior: Will the old habits return or die? Journal of Business Research, 117, 280-283. https://doi.org/10.1016/j.jbusres.2020.05.059

Tang, C. H., Chin, C. Y., \& Lee, Y. H. (2021). Coronavirus disease outbreak and supply chain disruption: Evidence from Taiwanese firms in China. Research in International Business and Finance, 56, 101355. https://doi. org/10.1016/j.ribaf.2020.101355

Thorgren, S., \& Williams, T. A. (2020). Staying alive during an unfolding crisis: How SMEs ward off impending disaster. Journal of Business Venturing Insights, 14, e00187. https://doi.org/10.1016/j.jbvi.2020.e00187

Useche Aguirre, M., Salazar Vázquez, F., Barragán Ramírez, C., \& Sánchez Salazar, P. (2020). Horizontes estratégicos empresariales en América Latina ante la pandemia generada por el COVID-19. SUMMA. Revista disciplinaria en ciencias económicas y sociales, 2(Especial), 59-86. https://doi.org/10.47666/summa.2.esp.07

Zahra, S. A. (2021). International entrepreneurship in the post Covid world. Journal of World Business, 56(1), 101143. https://doi.org/10.1016/j.jwb.2020.101143 